Volume 24 (2018) 107-128

DOI: $10.24330 /$ ieja.440235

\title{
UNIQUENESS OF DECOMPOSITION, FACTORISATIONS, $G$-GROUPS AND POLYNOMIALS
}

\author{
Alberto Facchini and Serap Şahinkaya \\ Received: 1 December 2017; Revised: 9 June 2018; Accepted: 9 June 2018 \\ Communicated by A. Çiğdem Özcan \\ Dedicated to our dear friend John Clark
}

\begin{abstract}
In this article, we present the classical Krull-Schmidt Theorem for groups, its statement for modules due to Azumaya, and much more modern variations on the theme, like the so-called weak Krull-Schmidt Theorem, which holds for some particular classes of modules. Also, direct product of modules is considered. We present some properties of the category of $G$-groups, a category in which Remak's results about the Krull-Schmidt Theorem for groups can be better understood. In the last section, direct-sum decompositions and factorisations in other algebraic structures are considered.
\end{abstract}

Mathematics Subject Classification (2010): 16D70, 16D90, 18E05

Keywords: Krull-Schmidt Theorem, indecomposable module, direct-sum decomposition, endomorphism ring, uniserial module, artinian module

\section{Introduction}

In this paper, we mainly present the classical Krull-Schmidt Theorem for groups and modules and some of its weak versions. According to the classical KrullSchmidt Theorem for modules, any module of finite composition length decomposes as a direct sum of indecomposable modules in an essentially unique way, that is, unique up to isomorphism of the indecomposable summands and a permutation of the summands. In Section 2, we present the historical background of that theorem.

Section 3 is devoted to commutative monoids, which provide the best algebraic tool to describe finite direct-sum decompositions of modules.

The first author was partially supported by Dipartimento di Matematica "Tullio Levi-Civita" of Università di Padova (Project BIRD163492/16 "Categorical homological methods in the study of algebraic structures" and Research program DOR1690814 "Anelli e categorie di moduli"). This survey is essentially based on an Algebra seminar that the first author gave on 9th February 2016 at the University of Edinburgh, entitled "Krull-Schmidt-Remak theorem, direct-sum decompositions of modules, direct-product decompositions of groups, $G$-groups". 
In 1975, Warfield proved that every finitely presented module over a serial ring is a finite direct sum of uniserial modules and posed a problem, essentially asking whether the Krull-Schimdt Theorem holds for finite direct sums of uniserial modules. More precisely, he asked whether the direct-sum decomposition of a finitely presented module over a serial ring into uniserial summands is unique up to isomorphism [37]. The negative answer to this question was given by the first author in 1996 [15]. He showed that even though the Krull-Schmidt Theorem does not hold for serial modules, a weak version of it holds not only for serial modules, but also for other classes of modules. Some of these classes of modules will be presented in Section 4.

It is natural to ask what happens when one considers arbitrary direct products of modules instead of arbitrary direct sums. In Section 5, we collect some results about direct product of modules and uniqueness of decomposition. In that section, we also present some properties of the category of $G$-groups, a framework in which the existence of the central automorphism in the Krull-Schmidt Theorem proved by Remak has a natural explanation.

All these results are strongly related to factorisation in a very general sense. Thus we present some results concerning factorisation into irreducible polynomials of non-commutative polynomials with coefficients in $\mathbb{Z}$, and also factorization of commutative polynomials with non-negative integral coefficients.

\section{Historical background of the Krull-Schmidt-Remak-Azumaya Theorem}

In this survey, rings will be associative rings $R$ with an identity, and modules will be unital right $R$-modules, unless otherwise stated.

In 1879, Frobenius and Stickelberger proved that any finite abelian group is a direct product of cyclic groups whose orders are powers of primes, and this powers of primes are uniquely determined by the group [23]. The "classical Krull-Schmidt Theorem for finite groups" was first stated in 1909 by Wedderburn [27], who published the following theorem.

Theorem 2.1. (Krull-Schmidt Theorem for finite groups) If a finite group G has two direct-product decompositions $G=G_{1} \times G_{2} \times \cdots \times G_{t}=H_{1} \times H_{2} \times \cdots \times H_{s}$ into indecomposables, then $t=s$ and there exist an automorphism $\varphi$ of $G$ and a permutation $\sigma$ of $\{1,2, \ldots, t\}$ such that $\varphi\left(G_{i}\right)=H_{\sigma(i)}$ for all indices $i=1,2, \ldots, t$.

The proof given by Wedderburn is not entirely convincing. It was Robert Erich Remak [32] who proved in his $\mathrm{PhD}$ dissertation (1911) that any two direct-product 
decompositions of a finite group into indecomposable factors are not only isomorphic, but also centrally isomorphic. More precisely, he proved the following theorem. Recall that a central automorphism of group $G$ is an automorphism of $G$ that induces the identity $G / \zeta(G) \rightarrow G / \zeta(G)$, where $\zeta(G)$ denotes the center of $G$.

Theorem 2.2. If a finite group $G$ has two direct-product decompositions into indecomposables $G=G_{1} \times G_{2} \times \cdots \times G_{t}=H_{1} \times H_{2} \times \cdots \times H_{s}$, then $t=s$ and there exist a central automorphism $\varphi$ of $G$ and a permutation $\sigma$ of $\{1,2, \ldots, t\}$ such that $\varphi\left(G_{i}\right)=H_{\sigma(i)}$ for all indices $i=1,2, \ldots, t$.

Otto Yulyevich Schmidt [33] then gave a simplified proof of Remak's results. These results were transfered to modules of finite length by Krull and Schimdt, getting a theorem that, in modern terminology, can be stated as follows.

Theorem 2.3. (The Krull-Schmidt Theorem) Let $R$ be a ring and $M$ be a module of finite length. Then there exists a decomposition

$$
M=M_{1} \oplus M_{2} \oplus \cdots M_{r}
$$

into indecomposable submodules $M_{i}$ of $M$. Moreover, if $M=N_{1} \oplus N_{2} \oplus \cdots N_{s}$ is another decomposition of $M$ into indecomposable modules, then $r=s$ and there exists a permutation $\sigma$ of $\{1, \cdots, r\}$ such that $M_{i} \cong N_{\sigma(i)}$ for every $i \in\{1, \cdots, r\}$.

Arbitrary modules do not decompose in an "essentially unique" way in general. Here is an example.

Example 2.4. [17] Let $R$ be a commutative integral domain with at least two distinct maximal ideals $M$ and $N$ that are not principal ideals. Then the morphism $M \oplus N \rightarrow R$, defined by $(x, y) \rightarrow x+y$, is an R-module epimorphism, which necessarily splits because $R$ is a projective $R$-module. The kernel of this morphism is isomorphic to $M \cap N$, so that there is a splitting short exact sequence $0 \rightarrow$ $M \cap N \rightarrow M \oplus N \rightarrow R \rightarrow 0$. Thus $M \oplus N$ is isomorphic to $R \oplus(M \cap N)$. But $M$ and $N$ are not principal ideals, so that they are not isomorphic to $R$. Therefore the two direct-sum decompositions are essentially different. Notice that $R$ is an $R$ module of Goldie dimension 1 since $R$ is a commutative integral domain. Thus $R$ and its submodules $M, N$ and $M \cap N$ are all modules of Goldie dimension 1, which proves that the two essentially different direct-sum decompositions are direct-sum decompositions into indecomposables.

Let $\Omega$ be a set. Recall that an $\Omega$-group is a pair $(H, \varphi)$, where $H$ is a group and $\varphi: \Omega \rightarrow \operatorname{End}(H)$ is a mapping. Krull [25] extended the results known for 
groups to the case of abelian operator groups with the ascending and descending chain conditions (operator groups $=\Omega$-groups). Notice that if $H$ is abelian, then $\operatorname{End}(H)$ is a ring, so that the mapping $\varphi: \Omega \rightarrow \operatorname{End}(H)$ extends uniquely to a ring homomorphism $\mathbb{Z}\langle\Omega\rangle \rightarrow \operatorname{End}(H)$, where $\mathbb{Z}\langle\Omega\rangle$ denotes the free ring with free set $\Omega$ of generators (=ring of non-commutative polynomials with coefficients in $\mathbb{Z}$ in the set of non-commuting indeterminates $\Omega$ ). Thus abelian $\Omega$-groups are exactly left $\mathbb{Z}\langle\Omega\rangle$-modules. The theory was subsequently further deepened by Schmidt [34].

Øystein Ore (Oslo, 1899-1968) unified the proofs from various categories: groups, abelian operator groups, rings and algebras. He showed that the theorem of Wedderburn holds for modular lattices with descending and ascending chain conditions.

The Krull-Schmidt Theorem was extended to the case of possibly infinite direct sums of modules with local endomorphism rings by Azumaya [6]. Notice that any indecomposable module of finite composition length has a local endomorphism ring (this is the so-called Fitting Lemma) and any module with a local endomorphism ring is necessarily indecomposable [4, p. 144].

Theorem 2.5. (Krull-Schmidt-Remak-Azumaya Theorem) Let $M$ be a module that is a direct sum of modules with local endomorphism rings. Then $M$ is a direct sum of indecomposable modules in an essentially unique way in the following sense. If

$$
M=\bigoplus_{i \in I} M_{i}=\bigoplus_{j \in J} N_{j}
$$

where all the submodules $M_{i}(i \in I)$ and $N_{j}(j \in J)$ are indecomposable, then there exists a bijection $\varphi: I \rightarrow J$ such that $M_{i} \cong N_{\varphi(i)}$ for every $i \in I$.

After this brief historical introduction, our aim now is to describe direct-sum decompositions of a module $M_{R}$ as a finite direct sum $M_{R}=M_{1} \oplus \cdots \oplus M_{n}$ of direct summands $M_{i}$. Several behaviours are possible. For instance:

- There can be uniqueness of direct-sum decomposition into indecomposables. This is the case described, for example, for modules that are direct sums of modules with local endomorphism rings (Krull-Schmidt-Remak-Azumaya).

- There are modules with a direct-sum decomposition into indecomposables, such that this decomposition is not unique in the sense of the Krull-SchmidtRemak-Azumaya Theorem, but there are only finitely many such direct-sum decompositions up to isomorphism. This happens, for instance, for torsionfree abelian groups of finite rank [26].

- For some classes of modules, direct-sum decompositions into indecomposables are not unique, but they enjoy some kind of regularity. 
- But, in general, there is no direct-sum decomposition into indecomposables, and no uniqueness as in Example 2.4.

\section{The reduced monoid $V(\mathcal{C})$}

The best way to describe finite direct-sum decompositions of a module $M_{R}$ is by making use of commutative monoids. A commutative monoid is a semigroup with a binary operation that is associative, commutative and has an identity element. All the monoids in this paper will be commutative and additive, that is, their operation will be denoted as an addition + , and their identity element will be denoted by 0 . For a commutative monoid $M$, let $U(M)$ denote the group of all $a \in M$ with an opposite $-a$ in $M$. A commutative additive monoid $M$ is reduced if $x, t \in M$ and $x+t=0$ implies $x=t=0$. That is, if $U(M)=0$. For every monoid $M$, the monoid $M_{\text {red }}:=M / U(M)$, whose elements are the cosets $m+U(M)$, is reduced.

We will look at classes of right $R$-modules as full subcategories of the category Mod- $R$ of all right $R$-modules. Let $\mathcal{C}$ be a category and let $V(\mathcal{C})$ denote a skeleton of $\mathcal{C}$, that is, a class of representatives of the objects of $\mathcal{C}$ modulo isomorphism. In order to avoid set-theoretical problems, in this survey we could consider only skeletally small categories, that is, categories $\mathcal{C}$ in which the class $\mathrm{Ob}(\mathcal{C})$ contains a set of representatives of the objects up to isomorphism. Equivalently, a category $\mathcal{C}$ is skeletally small if it has a skeleton whose class of objects is a set. For every object $A$ in $\mathcal{C}$, there is a unique object $\langle A\rangle$ in $V(\mathcal{C})$ isomorphic to $A$. Thus there is a mapping $\mathrm{Ob}(\mathcal{C}) \rightarrow V(\mathcal{C}), A \mapsto\langle A\rangle$, that associates to every object $A$ of $\mathcal{C}$ the unique object $\langle A\rangle$ in $V(\mathcal{C})$ isomorphic to $A$. Assume that a product $A \times B$ exists in $\mathcal{C}$ for every pair $A, B$ of objects of $\mathcal{C}$. Define an addition + in $V(\mathcal{C})$ by $A+B:=\langle A \times B\rangle$ for every $A, B \in V(\mathcal{C})$. If the category $\mathcal{C}$ is not skeletally small, we have that its skeleton $V(\mathcal{C})$ is a class that is not a set. This class becomes therefore a large monoid, that is, a monoid that is not a set but a class, like in the next lemma.

Lemma 3.1. Let $\mathcal{C}$ be a category with a terminal object and in which a product $A \times B$ exists for every pair $A, B$ of objects of $\mathcal{C}$. Then $V(\mathcal{C})$ is a large reduced commutative monoid.

It is easy to prove that the Krull-Schmidt property holds in the additive category $\mathcal{C}$ if and only if the monoid $V(\mathcal{C})$ is a free monoid, that is, isomorphic to the direct sum $\mathbb{N}^{(I)}$ for some class $I$.

An element $u$ of a commutative monoid $M$ is called an order-unit in $M$ if, for every $x \in M$, there exists an integer $n \geq 0$ such that $x \leq n u$. Here $\leq$ denotes 
the algebraic preorder on the commutative monoid $M$, that is, the reflexive and transitive relation $\leq$ on $M$ defined, for every $x, y \in M$, by $x \leq y$ if there exists $z \in M$ with $x+z=y$. Hence $u$ is an order-unit in $M$ if and only if, for every $x \in M$, there exist an integer $n \geq 0$ and an element $y \in M$ such that $x+y=n u$. It is now easy to define the category of commutative monoids with order-unit. Its objects are the pairs $(M, u)$, where $M$ is any commutative monoid and $u \in M$ is an order-unit. Its morphisms $f:(M, u) \rightarrow\left(M^{\prime}, u^{\prime}\right)$ are the monoid morphisms $f: M \rightarrow M^{\prime}$ such that $f(u)=u^{\prime}$.

For any ring $R$, let $\mathcal{C}$ be the class of all finitely generated projective right $R$ modules. We will denote by $V(R)$ the monoid $V(\mathcal{C})$. Then $V(R)$ is a commutative reduced monoid with order-unit $\left\langle R_{R}\right\rangle$.

The following theorem was first proved by Bergman for finitely generated monoids with order-unit [7, Theorems 6.2 and 6.4]. Then it was extended by Bergman and Dicks to arbitrary monoids with order-unit $[8$, p. 315]. Recall that a ring $R$ is hereditary if all its right ideals and all its left ideals are projective modules.

Theorem 3.2. $[7,8]$ Let $k$ be a field and let $(M, u)$ be a commutative reduced monoid with order-unit. Then there exists a hereditary $k$-algebra $R$ such that $(M, u)$ and $\left(V(R),\left\langle R_{R}\right\rangle\right)$ are isomorphic monoids with order-unit.

Corollary 3.3. Let $k$ be a field and let $M$ be a commutative reduced monoid. Then there exists a class $\mathcal{C}$ of finitely generated projective right modules over a hereditary $k$-algebra $R$ such that $M \cong V(\mathcal{C})$.

\section{Weak Krull-Schmidt Theorem}

Let $R$ be any ring. In this section, we will see what happens for some very special classes of right $R$-modules that are not direct sums of modules $M_{i}$ with local endomorphism ring $\operatorname{End}\left(M_{i}\right)$. A right $R$-module $M_{R}$ is uniserial if its lattice of submodules is linearly ordered, that is, if for any submodules $A, B$ of $M_{R}$ either $A \subseteq B$ or $B \subseteq A$, and it is serial if it is a direct sum of uniserial modules.

The endomorphism ring of a uniserial module has at most two maximal right (left) ideals, as the following theorem shows.

Theorem 4.1. [15] Let $U_{R}$ be a non-zero uniserial module over a ring $R, E:=$ $\operatorname{End}\left(U_{R}\right)$ its endomorphism ring, $I:=\{f \in E \mid f$ is not injective $\}$ and $K:=\{f \in$ $E \mid f$ is not surjective $\}$. Then $I$ and $K$ are two two-sided completely prime ideals of $E$, and every proper right ideal of $E$ and every proper left ideal of $E$ is contained either in $I$ or in $K$. Moreover, 
(a) either $E$ is a local ring with maximal ideal $I \cup K$, or

(b) $E / I$ and $E / K$ are division rings, and $E / J(E) \cong E / I \times E / K$.

In 1975, Warfield proved that every finitely presented module over a serial ring is a finite direct sum of uniserial modules, and asked whether the direct decomposition of a finitely presented module into uniserial summands is unique up to isomorphism [37]. Warfield's question was answered completely in [15], by showing that, although there exist serial rings for which the Krull-Schmidt Theorem does not hold for finitely presented modules, it is possible to prove a weak form of the Krull-Schmidt Theorem. Facchini's weak form of the Krull-Schmidt Theorem holds only for finite direct sums of uniserial modules, not for infinite ones. In order to state the theorem, we need the concepts of monogeny class and epigeny class of a module.

Two right $R$-modules $U$ and $V$ are said to have

(1) the same monogeny class, denoted $[U]_{m}=[V]_{m}$, if there exist a monomorphism $U \rightarrow V$ and a monomorphism $V \rightarrow U$;

(2) the same epigeny class, denoted $[U]_{e}=[V]_{e}$, if there exist an epimorphism $U \rightarrow V$ and an epimorphism $V \rightarrow U$.

The weak Krull-Schmidt Theorem for finite families of uniserial modules can be formulated as follows:

Theorem 4.2. (Weak Krull-Schmidt Theorem [15]) Let $U_{1}, \ldots, U_{n}, V_{1}, \ldots, V_{t}$ be $n+t$ non-zero uniserial right modules over a ring $R$. Then the direct sums $U_{1} \oplus \cdots \oplus U_{n}$ and $V_{1} \oplus \cdots \oplus V_{t}$ are isomorphic $R$-modules if and only if $n=t$ and there exist two permutations $\sigma$ and $\tau$ of $\{1,2, \ldots, n\}$ such that $\left[U_{i}\right]_{m}=\left[V_{\sigma(i)}\right]_{m}$ and $\left[U_{i}\right]_{e}=\left[V_{\tau(i)}\right]_{e}$ for every $i=1,2, \ldots, n$.

Remark 4.3. In [21], Zahra Nazemian and the first author have studied the factorisations $A=A_{1} \ldots A_{n}$ of a right ideal $A$ of a not-necessarily commutative ring $R$ as a product of right ideals $A_{1}, \ldots, A_{n}$, with $R / A \cong R / A_{1} \oplus \cdots \oplus R / A_{n}$ canonically and the right modules $R / A_{1}, \ldots, R / A_{n}$ uniserial. The main example of such a factorisation is the factorization into powers of prime ideals for non-zero ideals of a Dedekind domain $R$.

Theorem 4.2 must be modified if we want it to hold for infinite families of uniserial modules [13, Theorem 4.9]. This was done by Př́lhoda, who proved the converse of [13, Theorem 4.7] in [29]. First recall a definition. A right $R$-module $U_{R}$ is said to be quasismall if, for every family $\left\{M_{i} \mid i \in I\right\}$ of $R$-modules such that $U$ is 
isomorphic to a direct summand of $\bigoplus_{i \in I} M_{i}$, there is a finite set $I^{\prime} \subseteq I$ such that $U_{R}$ is isomorphic to a direct summand of $\bigoplus_{i \in I^{\prime}} M_{i}$.

Theorem 4.4. [29, Theorem 2.6] Let $\left\{U_{i} \mid i \in I\right\}$ and $\left\{V_{j} \mid j \in J\right\}$ be nonempty families of non-zero uniserial modules. Let $I^{\prime}=\left\{i \in I \mid U_{i}\right.$ is quasismall $\}$ and $J^{\prime}=\left\{j \in J \mid V_{j}\right.$ is quasismall $\}$. Then $\bigoplus_{i \in I} U_{i} \cong \bigoplus_{j \in J} V_{j}$ if and only if there exist a bijection $\sigma: I \rightarrow J$ and a bijection $\tau: I^{\prime} \rightarrow J^{\prime}$ such that $\left[U_{i}\right]_{m}=\left[V_{\sigma(i)}\right]_{m}$ for every $i \in I$ and $\left[U_{i}\right]_{e}=\left[V_{\tau(i)}\right]_{e}$ for every $i \in I^{\prime}$.

If a uniserial module $U$ has local endomorphism ring, then any direct summand of a direct sum $U^{(I)}$ of copies of $U$ is a direct sum of copies of $U$, because any uniserial module is $\sigma$-small (a module is $\sigma$-small if it is countable ascending union of small submodules) and one can use [16, Theorem 2.52].

Theorem 4.5. [30, Theorem 1.1] Let $U$ be a non-zero uniserial right module over a ring $R$. Then:

(1) If $g f \neq 0$ for every monomorphism $f: U \rightarrow U$ and every epimorphism $g: U \rightarrow U$, then every direct summand of a direct sum $U^{(I)}$ of copies of $U$ is a direct sum of copies of $U$.

(2) If $U$ is quasismall and there exist a monomorphism $f: U \rightarrow U$ and an epimorphism $g: U \rightarrow U$ such that $g f=0$, then every direct summand of a direct sum $U^{(I)}$ of copies of $U$ is isomorphic to $U^{(J)} \oplus V^{(K)}$, where $J$ and $K$ are suitable sets and $V$ is the unique uniserial module in the same monogeny class of $U$ that is not quasismall.

(3) If $U$ is not quasismall, then every direct summand of a direct sum $U^{(I)}$ of copies of $U$ is a direct sum of copies of $U$.

Recall that a right module over a ring $R$ is cyclically presented if it is isomorphic to $R / a R$ for some element $a \in R$. For any ring $R$, the endomorphism ring $\operatorname{End}_{R}(R / a R)$ of a non-zero cyclically presented module $R / a R$ is isomorphic to $E / a R$, where $E:=\{r \in R \mid r a \in a R\}$ is the idealizer of $a R$. The following theorem is proved in $[2$, Theorem 2.1].

Theorem 4.6. Let a be a non-zero non-invertible element of an arbitrary local ring $R$, let $E$ be the idealizer of $a R$, and let $E / a R$ be the endomorphism ring of the cyclically presented right $R$-module $R / a R$. Set $I:=\{r \in R \mid r a \in a J(R)\}$ and $K:=J(R) \cap E$. Then $I$ and $K$ are two two-sided completely prime ideals of $E$ containing aR, the union $(I / a R) \cup(K / a R)$ is the set of all non-invertible elements of $E / a R$, and every proper right ideal of $E / a R$ and every proper left ideal of $E / a R$ 
is contained either in $I / a R$ or in K/aR. Moreover, exactly one of the following two conditions holds:

(a) Either $I$ and $K$ are comparable (that is, $I \subseteq K$ or $K \subseteq I$ ), in which case $E / a R$ is a local ring, or

(b) $I$ and $K$ are not comparable, and in this case $E / I$ and $E / K$ are division rings, $J(E / a R)=(I \cap K) / a R$, and $(E / a R) / J(E / a R)$ is canonically isomorphic to the direct product $E / I \times E / K$.

For any ring $R$, let $U(R)$ denote the group of all invertible elements of $R$. If $R / a R$ and $R / b R$ are cyclically presented modules over a local ring $R$, we say that $R / a R$ and $R / b R$ have the same lower part, and write $[R / a R]_{l}=[R / b R]_{l}$, if there exist $u, v \in U(R)$ and $r, s \in R$ with $a u=r b$ and $b v=s a$. (It is possible to prove that two cyclically presented modules over a local ring have the same lower part if and only if their Auslander-Bridger transposes have the same epigeny class.)

Theorem 4.7. (Weak Krull-Schmidt Theorem for Cyclically Presented Modules) Let $a_{1}, \ldots, a_{n}, b_{1}, \ldots, b_{t}$ be $n+t$ non-invertible elements of a local ring $R$. Then the direct sums $R / a_{1} R \oplus \cdots \oplus R / a_{n} R$ and $R / b_{1} R \oplus \cdots \oplus R / b_{t} R$ are isomorphic right $R$-modules if and only if $n=t$ and there exist two permutations $\sigma, \tau$ of $\{1,2, \ldots, n\}$ such that $\left[R / a_{i} R\right]_{l}=\left[R / b_{\sigma(i)} R\right]_{l}$ and $\left[R / a_{i} R\right]_{e}=\left[R / b_{\tau(i)} R\right]_{e}$ for every $i=1,2, \ldots, n$.

The Weak Krull-Schmidt Theorem for cyclically presented modules has an immediate consequence as far as equivalence of matrices is concerned. Recall that two $m \times n$ matrices $A$ and $B$ with entries in a ring $R$ are said to be equivalent matrices, denoted $A \sim B$, if there exist an $m \times m$ invertible matrix $P$ and an $n \times n$ invertible matrix $Q$ with entries in $R$ (that is, matrices invertible in the rings $M_{m}(R)$ and $M_{n}(R)$, respectively) such that $B=P A Q$. We denote by $\operatorname{diag}\left(a_{1}, \ldots, a_{n}\right)$ the $n \times n$ diagonal matrix whose $(i, i)$ entry is $a_{i}$ and whose other entries are zero.

Remark 4.8. If $R$ is a commutative local ring and $a_{1}, \ldots, a_{n}, b_{1}, \ldots, b_{n}$ are elements of $R$, then $\operatorname{diag}\left(a_{1}, \ldots, a_{n}\right) \sim \operatorname{diag}\left(b_{1}, \ldots, b_{n}\right)$ if and only if there exists a permutation $\sigma$ of $\{1,2, \ldots, n\}$ with $a_{i}$ and $b_{\sigma(i)}$ associates for every $i=$ $1,2, \ldots, n$. Here $a, b \in R$ are associates if they generate the same principal ideal of $R$. Let's prove this. Assume $R$ commutative and local, and $\operatorname{diag}\left(a_{1}, \ldots, a_{n}\right) \sim$ $\operatorname{diag}\left(b_{1}, \ldots, b_{n}\right)$. Then $R / a_{1} R \oplus \cdots \oplus R / a_{n} R \cong R / b_{1} R \oplus \cdots \oplus R / b_{t} R$. Now the endomorphism ring of $R / a_{i} R$ is the ring $R / a_{i} R$, because $R$ is commutative, and therefore the endomorphism ring of each $R / a_{i} R$ is local, because $R$ is local. Similarly for the modules $R / b_{j} R$. Hence the Krull-Schmidt-Remak-Azumaya Theorem 
implies that there exists a permutation $\sigma$ of $\{1,2, \ldots, n\}$ with $R / a_{i} R \cong R / b_{\sigma(i)} R$ for every $i=1,2, \ldots, n$. Taking the annihilators of these isomorphic cyclic modules, we find that $a_{i} R=b_{\sigma(i)} R$ for every $i=1,2, \ldots, n$. Hence $a_{i}$ and $b_{\sigma(i)}$ are associates.

If the ring $R$ is local, but not-necessarily commutative, we have the following result.

Proposition 4.9. Let $a_{1}, \ldots, a_{n}, b_{1}, \ldots, b_{n}$ be elements of a local ring $R$. Then $\operatorname{diag}\left(a_{1}, \ldots, a_{n}\right) \sim \operatorname{diag}\left(b_{1}, \ldots, b_{n}\right)$ if and only if there exist two permutations $\sigma, \tau$ of $\{1,2, \ldots, n\}$ with

$$
\left[R / a_{i} R\right]_{l}=\left[R / b_{\sigma(i)} R\right]_{l} \quad \text { and } \quad\left[R / a_{i} R\right]_{e}=\left[R / b_{\tau(i)} R\right]_{e}
$$

for every $i=1,2, \ldots, n$.

As far as infinite direct sums are concerned, the case of cyclically presented modules over local rings is much simpler than that of uniserial modules [3]. The reason for this is that cyclically presented modules are finitely generated, hence small, so the pathology of non-quasismall modules can not appear in this setting.

Thus we have seen that, similarly to the endomorphism ring of a uniserial module over an arbitrary ring, the endomorphism ring of a cyclically presented module over a local ring also has at most two maximal right ideals (Theorem 4.6). By this fact, it is not surprising that there is an analogy between the behaviour of a direct sum of uniserial modules over arbitrary rings and the behaviour of a direct sum of cyclically presented modules over local rings. Therefore it makes sense to try to see which further parts of the theory of uniserial modules also hold for cyclically presented modules over local rings. Here are some very natural questions.

(1) Does the weak Krull-Schmidt Theorem hold for a direct sum of infinitely many cyclically presented modules over local rings?

(2) Is every direct summand of a direct sum of cyclically presented modules over a local ring a direct sum of cyclically presented modules?

(3) Is every direct summand of a direct sum of finitely many cyclically presented modules over a local ring a direct sum of cyclically presented modules?

The answer to the first question was given in [3, Theorem 3.1]. The answer to the second question is negative. To this end, there is an example given by Puninski in [31, Proposition 8.1]. For the third question, there exist countably generated indecomposable relatively divisible projective modules over local rings that are not direct sums of cyclically presented modules, and relatively divisible projective 
modules over local rings that are not direct sums of indecomposable modules. But, according to [36, Corollary 2], every relatively divisible projective module over a commutative local ring is a direct sum of cyclically presented modules.

Let us pass to consider another class of modules. For a right module $A_{R}$ over a ring $R$, let $E\left(A_{R}\right)$ denote the injective envelope of $A_{R}$. We say that two modules $A_{R}$ and $B_{R}$ have the same upper part, and write $\left[A_{R}\right]_{u}=\left[B_{R}\right]_{u}$, if there exist a homomorphism $\varphi: E\left(A_{R}\right) \rightarrow E\left(B_{R}\right)$ and a homomorphism $\psi: E\left(B_{R}\right) \rightarrow E\left(A_{R}\right)$ such that $\varphi^{-1}\left(B_{R}\right)=A_{R}$ and $\psi^{-1}\left(A_{R}\right)=B_{R}$.

Now we need a very standard technique of homological algebra that allows to extend a morphism between two modules to their injective resolutions. Let us present it. Assume that $E_{0}, E_{1}, E_{0}^{\prime}, E_{1}^{\prime}$ are indecomposable injective right modules over a ring $R$, and that $\varphi: E_{0} \rightarrow E_{1}, \varphi^{\prime}: E_{0}^{\prime} \rightarrow E_{1}^{\prime}$ are two right $R$-module morphisms. A morphism $f: \operatorname{ker} \varphi \rightarrow \operatorname{ker} \varphi^{\prime}$ extends to a morphism $f_{0}: E_{0} \rightarrow E_{0}^{\prime}$. Now $f_{0}$ induces a morphism $\widetilde{f}_{0}: E_{0} / \operatorname{ker} \varphi \rightarrow E_{0}^{\prime} / \operatorname{ker} \varphi^{\prime}$, which extends to a morphism $f_{1}: E_{1} \rightarrow E_{1}^{\prime}$. Thus we get a commutative diagram with exact rows

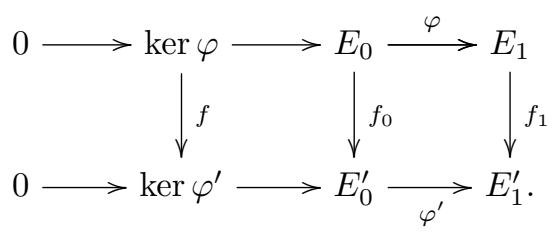

The morphisms $f_{0}$ and $f_{1}$ are not uniquely determined by $f$.

Theorem 4.10. Let $E_{0}$ and $E_{1}$ be indecomposable injective right modules over a ring $R$, and let $\varphi: E_{0} \rightarrow E_{1}$ be a non-zero non-injective morphism. Let $S:=$ $\operatorname{End}_{R}(\operatorname{ker} \varphi)$ denote the endomorphism ring of $\operatorname{ker} \varphi$. Set $I:=\{f \in S \mid$ the endomorphism $f$ of $\operatorname{ker} \varphi$ is not a monomorphism $\}$ and $K:=\{f \in S \mid$ the endomorphism $f_{1}$ of $E_{1}$ is not a monomorphism $\}=\left\{f \in S \mid \operatorname{ker} \varphi \subset f_{0}^{-1}(\operatorname{ker} \varphi)\right\}$. Then $I$ and $K$ are two two-sided completely prime ideals of $S$, and every proper right ideal of $S$ and every proper left ideal of $S$ is contained either in $I$ or in $K$. Moreover, exactly one of the following two conditions holds:

(a) Either $I$ and $K$ are comparable (that is, $I \subseteq K$ or $K \subseteq I$ ), in which case $S$ is a local ring with maximal ideal $I \cup K$, or

(b) $I$ and $K$ are not comparable, and in this case $S / I$ and $S / K$ are division rings and $S / J(S) \cong S / I \times S / K$.

The endomorphism ring of the kernel of a morphism between indecomposable injective modules has the same structure as the endomorphism ring of a uniserial 
module or a cyclically presented module over a local ring. More precisely, the endomorphism ring of the kernel of a morphism between two indecomposable injective modules is either local or has two maximal ideals, the kernel is determined up to isomorphism by its monogeny class and its upper part, and a weak form of the Krull-Schmidt Theorem also holds for direct sums of these kernels, as the following theorem shows.

Theorem 4.11. (Weak Krull-Schmidt Theorem for kernels of morphisms between indecomposable injective modules, [19, Theorem 2.7] and [14]) Let $\varphi_{i}: E_{i, 0} \rightarrow$ $E_{i, 1}(i=1,2, \ldots, n)$ and $\varphi_{j}^{\prime}: E_{j, 0}^{\prime} \rightarrow E_{j, 1}^{\prime}(j=1,2, \ldots, t)$ be $n+t$ non-injective morphisms between indecomposable injective right modules $E_{i, 0}, E_{i, 1}, E_{j, 0}^{\prime}, E_{j, 1}^{\prime}$ over an arbitrary ring $R$. Then the direct sums $\oplus_{i=0}^{n} \operatorname{ker} \varphi_{i}$ and $\oplus_{j=0}^{t} \operatorname{ker} \varphi_{j}^{\prime}$ are isomorphic $R$-modules if and only if $n=t$ and there exist two permutations $\sigma, \tau$ of $\{1,2, \ldots, n\}$ such that $\left[\operatorname{ker} \varphi_{i}\right]_{m}=\left[\operatorname{ker} \varphi_{\sigma(i)}^{\prime}\right]_{m}$ and $\left[\operatorname{ker} \varphi_{i}\right]_{u}=\left[\operatorname{ker} \varphi_{\tau(i)}^{\prime}\right]_{u}$ for every $i=1,2, \ldots, n$.

There are also some other classes of modules that satisfy the Weak Krull-Schmidt Theorem. One is the class of couniformly presented modules [20]. It generalizes the class of cyclically presented modules over a local ring. An $R$-module $M$ is said to be couniform if it has dual Goldie dimension 1, that is, it is nonzero and the sum of any two proper submodules of $M_{R}$ is a proper submodule of $M_{R}$. An $R$-module $M$ is couniformly presented if it is non-zero and there exists an exact sequence

$$
0 \rightarrow C_{R} \rightarrow P_{R} \rightarrow M_{R} \rightarrow 0
$$

with both $C_{R}$ and $P_{R}$ couniform and $P_{R}$ projective. Under these hypotheses, the exact sequence (2) is called a couniform presentation of the couniformly presented module $M_{R}$.

Theorem 4.12. (Weak Krull-Schmidt Theorem for couniformly presented modules [20]) Let $M_{1}, \ldots, M_{n}, N_{1}, \ldots, N_{t}$ be $n+t$ couniformly presented right $R$ modules. Then the direct sums $M_{1} \oplus \cdots \oplus M_{n}$ and $N_{1} \oplus \cdots \oplus N_{t}$ are isomorphic R-modules if and only if $n=t$ and there exist two permutations $\sigma$ and $\tau$ of $\{1,2, \ldots, n\}$ such that $\left[M_{i}\right]_{l}=\left[N_{\sigma(i)}\right]_{l}$ and $\left[M_{i}\right]_{e}=\left[N_{\tau(i)}\right]_{e}$ for every $i=1,2, \ldots, n$.

A similar behaviour, as far as direct-sum decompositions are concerned, takes place for the short exact sequences

$$
0 \rightarrow A_{R} \rightarrow B_{R} \rightarrow C_{R} \rightarrow 0,
$$

with $A_{R}$ and $C_{R}$ uniserial modules. The endomorphism ring of such a sequence in the category of all short exact sequences has at most four maximal ideals, and 
the isomorphism types of these sequences (3) are described by four invariants $[B]_{m, l},[B]_{e, l},[B]_{m, u},[B]_{e, u}[10,11]$.

We conclude this section by describing one of the general patterns that allow to treat all the previous examples at the same time. Let $\mathcal{C}$ be a full subcategory of the category Mod- $R$ for some ring $R$ and assume that every object of $\mathcal{C}$ is an indecomposable right $R$-module. Define a completely prime ideal $\mathcal{P}$ of $\mathcal{C}$ as an assignment of a subgroup $\mathcal{P}(A, B)$ of the additive abelian group $\operatorname{Hom}_{R}(A, B)$ to every pair $(A, B)$ of objects of $\mathcal{C}$, with the following two properties: (1) for every $A, B, C \in \operatorname{Ob}(\mathcal{C})$, every $f: A \rightarrow B$ and every $g: B \rightarrow C$, one has that $g f \in \mathcal{P}(A, C)$ if and only if either $f \in \mathcal{P}(A, B)$ or $g \in \mathcal{P}(B, C)$; (2) $\mathcal{P}(A, A)$ is a proper subgroup of $\operatorname{Hom}_{R}(A, A)$ for every object $A \in \mathrm{Ob}(\mathcal{C})$. Let $\mathcal{P}$ be a completely prime ideal of $\mathcal{C}$. If $A, B$ are objects of $\mathcal{C}$, we say that $A$ and $B$ have the same $\mathcal{P}$ class, and write $[A]_{\mathcal{P}}=[B]_{\mathcal{P}}$, if $\mathcal{P}(A, B) \neq \operatorname{Hom}_{R}(A, B)$ and $\mathcal{P}(B, A) \neq \operatorname{Hom}_{R}(B, A)$.

Theorem 4.13. [22] Let $\mathcal{C}$ be a full subcategory of Mod- $R$ and $\mathcal{P}, \mathcal{Q}$ be two completely prime ideals of $\mathcal{C}$. Assume that all objects of $\mathcal{C}$ are indecomposable right $R$-modules and that, for every $A \in \mathrm{Ob}(\mathcal{C}), f: A \rightarrow A$ is an automorphism of $A$ if and only if $f \notin \mathcal{P}(A, A) \cup \mathcal{Q}(A, A)$. Then, for every $A_{1}, \ldots, A_{n}, B_{1}, \ldots, B_{t} \in \mathrm{Ob}(\mathcal{C})$, the modules $A_{1} \oplus \cdots \oplus A_{n}$ and $B_{1} \oplus \cdots \oplus B_{t}$ are isomorphic if and only if $n=t$ and there exist two permutations $\sigma, \tau$ of $\{1,2, \ldots, n\}$ such that $\left[A_{i}\right]_{\mathcal{P}}=\left[B_{\sigma(i)}\right]_{\mathcal{P}}$ and $\left[A_{i}\right]_{\mathcal{Q}}=\left[B_{\tau(i)}\right]_{\mathcal{Q}}$ for all $i=1, \ldots, n$.

A further remark: for the classes $\mathcal{C}$ of modules described so far, the fact that the weak form of the Krull-Schmidt Theorem holds can be described by saying that the corresponding monoid $V(\mathcal{C})$ is a subdirect product of two free monoids.

\section{Direct products of modules whose endomorphism rings have at most two maximal ideals}

In the previous section, we have seen that the Weak Krull-Schmidt Theorem holds not only for uniserial modules, but also for cyclically presented modules over a local ring $R$, for kernels of morphisms between indecomposable injective modules, for couniformly presented modules, and more generally, for a number of classes of modules with at most two maximal right ideals. In this section, we will see that a similar result can hold not only for direct sums, but also for direct products of modules.

In order to present the main result in the most general setting, that of modules whose endomorphism rings have at most two maximal right ideals, we begin from the Weak Krull-Schimidt Theorem for direct products of uniserial modules. 
Theorem 5.1. [1] Let $\left\{U_{i} \mid i \in I\right\}$ and $\left\{V_{j} \mid j \in J\right\}$ be two families of uniserial modules over an arbitrary ring $R$. Assume that there exist two bijections $\sigma, \tau: I \rightarrow J$ such that $\left[U_{i}\right]_{m}=\left[V_{\sigma(i)}\right]_{m}$ and $\left[U_{i}\right]_{e}=\left[V_{\tau(i)}\right]_{e}$ for every $i \in I$. Then $\prod_{i \in I} U_{i} \cong$ $\prod_{j \in J} V_{j}$.

A full subcategory $\mathcal{C}$ of Mod- $R$ is said to satisfy Condition (DSP) (direct summand property) if whenever $A, B, C, D$ are right $R$-modules with $A \oplus B \cong C \oplus D$ and $A, B, C \in \mathrm{Ob}(\mathcal{C})$, then also $D \in \mathrm{Ob}(\mathcal{C})$.

Theorem 5.2. [1] Let $\mathcal{C}$ be a full subcategory of $\operatorname{Mod}-R$ in which all objects are indecomposable right $R$-modules and let $\mathcal{P}, \mathcal{Q}$ be two completely prime ideals of $\mathcal{C}$ with the property that, for every $A \in \mathrm{Ob}(\mathcal{C}), f: A \rightarrow A$ is an automorphism if and only if $f \notin \mathcal{P}(A, A) \cup \mathcal{Q}(A, A)$. Assume that $\mathcal{C}$ satisfies Condition (DSP). Let $\left\{A_{i} \mid i \in I\right\}$ and $\left\{B_{j} \mid j \in J\right\}$ be two families of objects of $\mathcal{C}$. Suppose that there exist two bijections $\sigma, \tau: I \rightarrow J$ such that $\left[A_{i}\right]_{\mathcal{P}}=\left[B_{\sigma(i)}\right]_{\mathcal{P}}$ and $\left[A_{i}\right]_{\mathcal{Q}}=\left[B_{\tau(i)}\right]_{\mathcal{Q}}$ for every $i \in I$. Then the $R$-modules $\prod_{i \in I} A_{i}$ and $\prod_{j \in J} B_{j}$ are isomorphic.

\section{The category of $G$-groups}

We can also find the same behaviour in other algebraic structures, not only in modules. We can find it, for instance, in groups, Lie algebras, $G$-groups, and so on. We begin with $G$-groups. The category of $G$-groups shows a behaviour that is a very pleasant combination of the behaviour of groups and that of left modules over a ring.

Let $G$ be a group. A (left) $G$-group is a pair $(H, \varphi)$, where $H$ is a group and $\varphi: G \rightarrow \operatorname{Aut}(H)$ is a group homomorphism. Equivalently, a $G$-group is a group $H$ endowed with a mapping $: G \times H \rightarrow H,(g, h) \mapsto g h$, called left scalar multiplication, such that

(a) $g\left(h h^{\prime}\right)=(g h)\left(g h^{\prime}\right)$

(b) $\left(g g^{\prime}\right) h=g\left(g^{\prime} h\right)$

(c) $1_{G} h=h$

for every $g, g^{\prime} \in G$ and every $h, h^{\prime} \in H$.

The notion of a $G$-group is classical. Sometimes $G$ is called an operator group on $H$ [35, Definition 8.1].

As an example of a $G$-group, let $\alpha_{g}: G \rightarrow G, \alpha_{g}: h \mapsto g h g^{-1}$, be the inner automorphism of $G$ determined by $g$, for every $g \in G$. Then there is a canonical group morphism $\alpha: G \rightarrow \operatorname{Aut}(G), g \mapsto \alpha_{g}$, which makes $G$ a $G$-group. It is called the regular $G$-group, exactly how the left $R$-module ${ }_{R} R$ is called the regular left $R$-module. 
The category $G$-Grp of $G$-groups has as objects all pairs $(H, \varphi)$, where $H$ is any group and $\varphi: G \rightarrow \operatorname{Aut}(H)$ is a group homomorphism. The morphisms in the category $G$-Grp are the group morphisms $f: H \rightarrow H^{\prime}$ such that $f(g h)=g f(h)$ for every $g \in G, h \in H$. The category $G$-Grp is a semiabelian category, in which the abelian objects are the objects $(H, \varphi)$ with $H$ an abelian group. We will see at the end of this section that the category $G$-Grp provides the proper setting to study direct product decompositions of a group $G$ as a direct product of finitely many indecomposable groups.

Remark 6.1. Instead of considering left $G$-groups, one can study right $G$-groups. A right $G$-group is defined as a pair $\left(H, \varphi^{\prime}\right)$, where $H$ is a group and $\varphi^{\prime}: G \rightarrow \operatorname{Aut}(H)$ is a group antihomomorphism. Equivalently, a right $G$-group is a group $H$ endowed with a mapping $\cdot: H \times G \rightarrow H,(h, g) \mapsto h g$, called right scalar multiplication, such that (a) becomes $\left(h h^{\prime}\right) g=(h g)\left(h^{\prime} g\right)$, and so on. We can thus construct the category of Grp-G of all right $G$-groups. But if $G^{\text {op }}$ denotes the opposite group of the group $G$, that is, the set $G$ with multiplication $(x, y) \mapsto y x$, then $G$ is isomorphic to $G^{\mathrm{op}}$ via the isomorphism $G \rightarrow G^{\mathrm{op}}, g \mapsto g^{-1}$. Now it is easily seen that the category $G$-Grp of left $G$-groups is isomorphic to the category $\mathbf{G r p}-G^{\text {op }}$ of right $G^{\text {op }}$-groups, and that Grp- $G$ is isomorphic to $G^{\mathrm{op}}$-Grp. But from the group isomorphism $G \rightarrow G^{\mathrm{op}}$, we also have that the categories $G-\mathbf{G r p}$ and $\mathbf{G r p}-G$ are isomorphic. So there is no need to introduce both left $G$-groups and right $G$-groups, because they form isomorphic categories, and therefore corresponding objects in this category isomorphism are indistinguishable from an algebraic point of view.

Notice the strict analogy between the categories $R$-Mod and $G$-Grp. Objects of $R$-Mod are all pairs $(H, \varphi)$, where $H$ is any abelian group and $\varphi: R \rightarrow \operatorname{End}(H)$ is a ring homomorphism. As we have already said, the regular $G$-group $(G, \alpha)$ plays, in the category $G$-Grp, a role pretty much similar to the role of the regular module ${ }_{R} R$ in the category $R$-Mod.

The automorphisms in $G$-Grp of the regular object $G$ are exactly the central automorphisms of $G$, that is, the automorphisms of $G$ that belong to the kernel of the canonical mapping $\operatorname{Aut}(G) \rightarrow \operatorname{Aut}(G / \zeta(G))$. Thus the automorphisms of $G$ in $G$-Grp are exactly the automorphisms of the group $G$ of the form $1+\varphi$ for some endomorphism $\varphi$ of $G$ with $\varphi(G) \subseteq \zeta(G)$. Here the sum of two endomorphisms $\varphi, \psi$ of a group $G$ is defined by $(\varphi+\psi)(g)=\varphi(g) \psi(g)$. The $\operatorname{sum} \varphi+\psi$ is an endomorphism of $G$ only when $[\varphi(G), \psi(G)]=1$. It is easy to see that if $\varphi$ is a group endomorphism of $G$ with $\varphi(G) \subseteq \zeta(G)$, then $1+\varphi$ is an automorphism of $G$ if and only if the restriction of $1+\varphi$ to $\zeta(G)$ is an automorphism of the abelian group $\zeta(G)$. 
The central automorphism $\varphi$ in the statement of Theorem 2.2, whose existence was proved by Remak, corresponds exactly to the fact that Remark, in the study of direct-product decompositions of a group $G$, was studying the decompositions of the regular object $G$ in the category $G$-Grp and not the decompositions of the group $G$ in the category Grp. Of course, he didn't know what a category is.

An interesting role in this setting is played by the full subcategory $\mathcal{C}_{G}$ of $G$ Grp consisting of all the objects $(H, \varphi)$ of $G$-Grp for which the image of the group homomorphism $\varphi: G \rightarrow \operatorname{Aut}(H)$ contains the group $\operatorname{Inn}(H)$ of all inner automorphisms of $H$ [18].

Another setting in which the Weak Krull-Schmidt Theorem holds in Group Theory is in the study of abelian normal subgroups of a group $G$ [5]. If $G$ is an arbitrary group and $H$ is an abelian normal subgroup of $G$, then the conjugation $\alpha: G \rightarrow \operatorname{Aut}(G)$ induces an action $\alpha: G \rightarrow \operatorname{Aut}(H)$ because $H$ is normal in $G$. If $\mathbb{Z}[G]$ is the group algebra of $G$ with integer coefficients, then the conjugation $\alpha: G \rightarrow \operatorname{Aut}(H)$ extends to a ring morphism $\mathbb{Z}[G] \rightarrow \operatorname{End}(H)$, so that $H$ turns out to be a left module over the ring $\mathbb{Z}[G]$. Hence Theorem 4.2 can be applied to this left module whenever $H$ is a direct sum of uniserial left $\mathbb{Z}[G]$-modules.

\section{Factorisation}

Everything we've seen until now corresponds, in a broad sense, to study factorisations of elements in suitable monoids, for instance in the commutative monoid $V(R)$. Let's see how what we have learned about direct-sum decompositions (directproduct decompositions) also holds for factorisations in other classes of monoids. For instance, it can be applied to factorisations of elements in a commutative domain $R$, because clearly factorisations of elements in an integral domain $R$ are exactly factorisations in the multiplicative monoid of $R$. We all know that a unique factorisation domain (UFD) is a commutative integral domain $R$ such that:

(i) $R$ is atomic, that is, every element $a \in R, a \neq 0$ and $a$ non-invertible, is a product of finitely many irreducible elements of $R$.

(ii) If $p_{1}, \ldots, p_{n}, q_{1}, \ldots, q_{m}$ are irreducible elements of $R$ and $p_{1} \ldots p_{n}=q_{1} \ldots q_{m}$, then $n=m$ and there exists a permutation $\sigma$ of $\{1,2, \ldots, n\}$ such that $p_{i}$ and $q_{\sigma(i)}$ are associates for every $i=1,2, \ldots, n$.

Thus an integral domain $R$ is a unique factorisation domain if and only if the multiplicative monoid $R \backslash\{0\}$ is isomorphic to the direct product of the abelian group $U(R)$ and a free commutative monoid $F$. 
Notice that, according to a very nice result by J. Coykendall and W. W. Smith [12]:

Theorem 7.1. A commutative integral domain $R$ is a UFD if and only if

(i) $R$ is atomic.

(ii) If $n \geq 2$ and $p_{1}, \ldots, p_{n}, q_{1}, \ldots, q_{n}$ are irreducible elements of $R$ and $p_{1} \ldots p_{n}=$ $q_{1} \ldots q_{n}$, then there exists a permutation $\sigma$ of $\{1,2, \ldots, n\}$ such that $p_{i}$ and $q_{\sigma(i)}$ are associates for every $i=1,2, \ldots, n$.

In an integral domain $R$, every prime element is irreducible. If $R$ is a UFD, the converse holds. More precisely, an integral domain $R$ is a UFD if and only if every irreducible is prime and $R$ satisfies the ascending chain condition on principal ideals, if and only if $R$ is atomic and every irreducible is prime.

Proposition 7.2. The following conditions are equivalent for two prime elements $a, b$ of a commutative integral domain $R$ :

(i) $a=b u$ for some invertible element $u \in R$.

(ii) $a R=b R$.

(iii) $R / a R \cong R / b R$.

(iv) $[R / a R]_{m}=[R / b R]_{m}$.

(v) $[R / a R]_{e}=[R / b R]_{e}$.

(vi) $[R / a R]_{l}=[R / b R]_{l}$.

Let's pass to consider, now, factorisation of polynomials into irreducible polynomials.

The case of commutative polynomials in commuting indeterminates is well known: the ring $\mathbb{Z}\left[x_{1}, \ldots, x_{n}\right]$ of all polynomials whose coefficients are in the ring of integers $\mathbb{Z}$ and with $x_{1}, \ldots, x_{n}$ commuting indeterminates is a UFD.

Let's see how the situation changes when we pass to the ring $\mathbb{Z}\left\langle x_{1}, \ldots, x_{n}\right\rangle$, the free ring on $n$ objects. The elements of $\mathbb{Z}\left\langle x_{1}, \ldots, x_{n}\right\rangle$ are non-commutative polynomials with coefficients in $\mathbb{Z}$ and with $x_{1}, \ldots, x_{n}$ non-commuting indeterminates. The ring $\mathbb{Z}\left\langle x_{1}, \ldots, x_{n}\right\rangle$ is atomic, in the sense that polynomials do factorise as a product of irreducible polynomials. The invertible elements in $\mathbb{Z}\left\langle x_{1}, \ldots, x_{n}\right\rangle$ are only 1 and -1 . But the factorisation $x(y x-2)=(x y-2) x$ in the ring $\mathbb{Z}\langle x, y\rangle$ shows that a polynomial in $\mathbb{Z}\left\langle x_{1}, \ldots, x_{n}\right\rangle$ does not necessarily factorise as a product of irreducible polynomials in a unique way up to the sign of the irreducible factors. Nevertheless the following theorem holds:

Theorem 7.3. (Brungs' Theorem [9]) Every polynomial in $R:=\mathbb{Z}\left\langle x_{1}, \ldots, x_{n}\right\rangle$ factorises as a product of irreducible polynomials. Moreover, if $p_{1}, \ldots, p_{n}, q_{1}, \ldots, q_{m}$ 
are irreducible polynomials in $R$ and $p_{1} \ldots p_{n}=q_{1} \ldots q_{m}$, then $n=m$ and there exists a permutation $\sigma$ of $\{1,2, \ldots, n\}$ such that $\left[R / p_{i} R\right]_{m}=\left[R / q_{\sigma(i)} R\right]_{m}$.

For instance, consider the factorisation $x(y x-2)=(x y-2) x$ in the ring $R=\mathbb{Z}\langle x, y\rangle$. We have that $[R /(x y-2) R]_{m}=[R /(y x-2) R]_{m}$, because left multiplications

$$
\lambda_{y}: R /(x y-2) R \rightarrow R /(y x-2) R \quad \text { and } \quad \lambda_{x}: R /(y x-2) R \rightarrow R /(x y-2) R
$$

are right $R$-module monomorphisms.

Let's pass to consider another interesting type of polynomials, those in the multiplicative monoid $\mathbb{N}_{0}[x]$, consisting of all polynomials in $\mathbb{Z}[x]$ whose integer coefficients are all $\geq 0$.

The multiplicative monoid $\mathbb{N}_{0}[x]$ is not a ring, it is a commutative semiring. The multiplicative monoid $\mathbb{N}_{0}[x] \backslash\{0\}$ is atomic, in the sense that all its elements are finite products of atoms, that is, polynomials irreducible in $\mathbb{N}_{0}[x] \backslash\{0\}$. The unique invertible element in $\mathbb{N}_{0}[x]$ is 1 . The next example shows that a polynomial in $\mathbb{N}_{0}[x]$ does not necessarily factorise as a product of irreducible polynomials in a unique way up to the order of the factors:

Example 7.4. From the theory of cyclotomic polynomials, we know that the factorisation of $x^{n}-1$ in the UFD $\mathbb{Q}[x]$ is $x^{n}-1=\prod_{d \mid n} \Phi_{d}(x)$, where $\Phi_{d}(x)$ is the $d$-th cyclotomic polynomial. Here

$\Phi_{1}(x)=x-1, \Phi_{2}(x)=x+1, \Phi_{3}(x)=x^{2}+x+1, \Phi_{4}(x)=x^{2}+1, \Phi_{5}(x)=$ $x^{4}+x^{3}+x^{2}+x+1, \Phi_{6}(x)=x^{2}-x+1$. Thus $x^{6}-1=\Phi_{1}(x) \Phi_{2}(x) \Phi_{3}(x) \Phi_{6}(x)=$ $(x-1)(x+1)\left(x^{2}+x+1\right)\left(x^{2}-x+1\right)$, so we have the factorisation

$$
x^{5}+x^{4}+x^{3}+x^{2}+x+1=(x+1)\left(x^{2}+x+1\right)\left(x^{2}-x+1\right)
$$

into irreducibles in $\mathbb{Q}[x]$. Multiplying the first factor and the last one in the factorisation (4), we get that $(x+1)\left(x^{2}-x+1\right)=x^{3}+1 \in \mathbb{N}_{0}[x]$ and, multiplying the last two factors, we get that $\left(x^{2}+x+1\right)\left(x^{2}-x+1\right)=x^{4}+x^{2}+1 \in \mathbb{N}_{0}[x]$. Thus we get two essentially different factorisations

$$
\left(x^{3}+1\right)\left(x^{2}+x+1\right)=(x+1)\left(x^{4}+x^{2}+1\right)
$$

of $x^{5}+x^{4}+x^{3}+x^{2}+x+1$ into irreducibles of $\mathbb{N}_{0}[x]$.

Thus factorisations into irreducibles in $\mathbb{N}_{0}[x]$ are not unique (but every polynomial in $\mathbb{N}_{0}[x]$ has only finitely many distinct factorisations into irreducibles). 
Example 7.4 can be applied to show that Krull-Schmidt fails in other categories. For instance, consider the category of finite partially ordered sets. This category has coproducts (disjoint unions), products (direct products with the componentwise order) and a terminal object 1 (the partially ordered set with one element), which is the identity with respect to product. Let $L=\{0,1\}$ denote the partially ordered set with two elements $0<1$. Then, for every $n \geq 0$, the direct product $L^{n}$ is a connected partially ordered set with $2^{n}$ elements, and its automorphism group is the symmetric group $S_{n}$. If we compute the identity (5), which is an identity in the semiring $\mathbb{N}_{0}[x]$, replacing $x$ with $L$ (and the natural number 1 with the partially ordered set 1), we get two essentially different direct-product decompositions of the partially ordered set $1 \dot{\cup} L \dot{\cup} L^{2} \dot{\cup} L^{3} \dot{\cup} L^{4} \dot{\cup} L^{5}$ into indecomposable partially ordered sets, that is, we get that

$$
\left(L^{3} \dot{\cup} 1\right) \times\left(L^{2} \dot{\cup} L \dot{\cup} 1\right) \cong(L \dot{\cup} 1) \times\left(L^{4} \dot{\cup} L^{2} \dot{\cup} 1\right) .
$$

This example, due to Nakayama and Hashimoto [24,28], shows that Krull-Schmidt fails in the category of finite partially ordered sets.

The possibility of applying the identity (4) in $\mathbb{N}_{0}[x]$ to get an isomorphism in the category of finite partially ordered sets is due to the fact that distributivity holds for partially ordered sets: $X \times(Y \dot{\cup} Z) \cong(X \times Y) \dot{\cup}(X \times Z)$. More generally, recall that a category $\mathcal{C}$ with finite products $(-) \times(-)$ and coproducts $(-)+(-)$ is called (finitary) distributive if, for any objects $X, Y, Z$ of $\mathcal{C}$, the canonical morphism

$$
X \times Y+X \times Z \rightarrow X \times(Y+Z)
$$

is an isomorphism. It is now easily seen that Nakayama and Hashimoto's technique can be applied to any distributive category.

\section{References}

[1] A. Alahmadi and A. Facchini, Direct products of modules whose endomorphism rings have at most two maximal ideals, J. Algebra, 435 (2015), 204-222.

[2] B. Amini, A. Amini and A. Facchini, Equivalence of diagonal matrices over local rings, J. Algebra, 320(3) (2008), 1288-1310.

[3] A. Amini, B. Amini and A. Facchini, Weak Krull-Schmidt for infinite direct sums of cyclically presented modules over local rings, Rend. Semin. Mat. Univ. Padova, 122 (2009), 39-54.

[4] F. W. Anderson and K. R. Fuller, Rings and Categories of Modules, New York, Springer-Verlag, 1974. 
[5] M. J. Arroyo Paniagua and A. Facchini, G-groups and biuniform abelian normal subgroups, Adv. Group Theory Appl., 2 (2016), 79-111.

[6] G. Azumaya, Corrections and supplementaries to my paper concerning KrullRemak-Schmidt's theorem, Nagoya Math. J., 1 (1950), 117-124.

[7] G. M. Bergman, Coproducts and some universal ring constructions, Trans. Amer. Math. Soc., 200 (1974), 33-88.

[8] G. M. Bergman and W. Dicks, Universal derivations and universal ring constructions, Pac. J. Math., 79 (1978), 293-337.

[9] H.-H. Brungs, Ringe mit eindeutiger Faktorzerlegung, J. Reine Angew. Math., 236 (1969), 43-66.

[10] F. Campanini, On a category of chains of modules whose endomorphism rings have at most $2 n$ maximal right ideals, Comm. Algebra, 46(5) (2018), 1971-1982.

[11] F. Campanini and A. Facchini, On a category of extensions whose endomorphism rings have at most four maximal ideals, to appear in "Advances in Rings and Modules, S. López-Permouth, J. K. Park, C. Roman and S. T. Rizvi Eds, Contemp. Math., 2018.

[12] J. Coykendall and W. W. Smith, On unique factorisation domains, J. Algebra, 332 (2011), 62-70.

[13] N. V. Dung and A. Facchini, Weak Krull-Schmidt for infinite direct sums of uniserial modules, J. Algebra, 193 (1997), 102-121.

[14] Ş. Ecevit, A. Facchini, and M. T. Koşan, Direct sums of infinitely many kernels, J. Aust. Math. Soc., 89(2) (2010), 199-214.

[15] A. Facchini, Krull-Schmidt fails for serial modules, Trans. Amer. Math. Soc., 348(11) (1996), 4561-4575.

[16] A. Facchini, Module Theory. Endomorphism Rings and Direct Sum Decompositions in Some Classes of Modules, Birkhäuser Verlag, Basel, 1998.

[17] A. Facchini, Direct-sum decompositions of modules with semilocal endomorphism rings, Bull. Math. Sci., 2(2) (2012), 225-279.

[18] A. Facchini and M. Altun-Özarslan, The Krull-Schmidt-Remak-Azumaya Theorem for G-groups, to appear in the proceedings of the Conference "Noncommutative rings and their applications, V", Lens 12-15 June 2017, Contemp. Math., 2018.

[19] A. Facchini, Ş. Ecevit and M. T. Koşan, Kernels of morphisms between indecomposable injective modules, Glasg. Math. J., 52(A) (2010), 69-82. 
[20] A. Facchini and N. Girardi, Couniformly presented modules and dualities, Advances in ring theory, Trends Math., Birkhâuser/Springer Basel AG, Basel, (2010), 149-164.

[21] A. Facchini and Z. Nazemian, Equivalence of some homological conditions for ring epimorphisms, to appear in J. Pure Appl. Algebra, 2018.

[22] A. Facchini and P. Př́hoda, The Krull-Schmidt theorem in the case two, Algebr. Represent. Theory, 14(3) (2011), 545-570.

[23] H. Frobenius and H. Stickelberger, Über Gruppen von vertauschbaren Elementen, J. Reine Angew. Math., 86 (1879), 217-262.

[24] J. Hashimoto, On direct product decomposition of partially ordered sets, Ann. of Math., 54(2) (1951), 315-318.

[25] W. Krull, Über verallgemeinerte endliche Abelsche Gruppen, Math. Z., 23(1) (1925), 161-196.

[26] E. L. Lady, Summands of finite rank torsion-free abelian groups, J. Algebra, 32 (1974), 51-52.

[27] J. H. Maclagan-Wedderburn, On the direct product in the theory of finite groups, Ann. of Math., 10(4) (1909), 173-176.

[28] T. Nakayama, and J. Hashimoto, On a problem of G. Birkhoff, Proc. Amer. Math. Soc., 1 (1950), 141-142.

[29] P. Př́hoda, A version of the weak Krull-Schmidt theorem for infinite direct sums of uniserial modules, Comm. Algebra, 34(4) (2006), 1479-1487.

[30] P. Př́hoda, $\operatorname{Add}(U)$ of a uniserial module, Comment. Math. Univ. Carolin., 47(3) (2006), 391-398.

[31] G. Puninski, Some model theory over a nearly simple uniserial domain and decompositions of serial modules, J. Pure Appl. Algebra, 163(3) (2001), 319337.

[32] R. E. Remak, Über die Zerlegung der endlichen Gruppen in indirekte unzerlegbare faktoren, dissertation, 1911.

[33] O. Y. Schmidt, Sur les produits directs, Bull. Soc. Math. France, 41 (1913), 161-164.

[34] O. Y. Schmidt, Über unendliche Gruppen mit endlicher Kette, Math. Z., 29 (1929), 34-41.

[35] M. Suzuki, Group Theory I, Springer-Verlag, Berlin-New York, 1982.

[36] R. B. Warfield, Purity and algebraic compactness for modules, Pacific J. Math., 28 (1969), 699-719. 
[37] R. B. Warfield, Serial rings and finitely presented modules, J. Algebra, 37 (1975), 187-222.

Alberto Facchini (Corresponding Author)

Dipartimento di Matematica Pura e Applicata

Università di Padova

35121 Padova, Italy

e-mail: facchini@math.unipd.it

Serap Şahinkaya

Department of Mathematics

Faculty of Science

Gebze Technical University

41400 Gebze, Kocaeli, Turkey

e-mail:ssahinkaya@gtu.edu.tr 